\title{
A!
}

This is an electronic reprint of the original article.

This reprint may differ from the original in pagination and typographic detail.

Möttönen, Mikko; Vartiainen, Juha; Pekola, Jukka

\section{Experimental determination of the Berry phase in a superconducting charge pump}

Published in:

Physical Review Letters

DOI:

10.1103/PhysRevLett.100.177201

Published: 28/04/2008

Document Version

Publisher's PDF, also known as Version of record

Please cite the original version:

Möttönen, M., Vartiainen, J., \& Pekola, J. (2008). Experimental determination of the Berry phase in a superconducting charge pump. Physical Review Letters, 100(17), 1-4. [177201].

https://doi.org/10.1103/PhysRevLett.100.177201

This material is protected by copyright and other intellectual property rights, and duplication or sale of all or part of any of the repository collections is not permitted, except that material may be duplicated by you for your research use or educational purposes in electronic or print form. You must obtain permission for any other use. Electronic or print copies may not be offered, whether for sale or otherwise to anyone who is not an authorised user. 


\title{
gs
}

\section{Experimental Determination of the Berry Phase in a Superconducting Charge Pump}

\author{
Mikko Möttönen, ${ }^{1,2, *}$ Juha J. Vartiainen, ${ }^{1}$ and Jukka P. Pekola ${ }^{1}$ \\ ${ }^{1}$ Low Temperature Laboratory, Helsinki University of Technology, P.O. Box 3500, 02015 TKK, Finland \\ ${ }^{2}$ Department of Engineering Physics/COMP, Helsinki University of Technology, P. O. Box 5100, 02015 TKK, Finland
}

(Received 7 November 2007; published 28 April 2008)

\begin{abstract}
We present the first measurements of the Berry phase in a superconducting Cooper pair pump. A fixed amount of Berry phase is accumulated to the quantum-mechanical ground state in each adiabatic pumping cycle, which is determined by measuring the charge passing through the device. The dynamic and geometric phases are identified and measured quantitatively from their different response when pumping in opposite directions. Our observations, in particular, the dependencies of the dynamic and geometric effects on the superconducting phase bias across the pump, agree with the basic theoretical model of coherent Cooper pair pumping.
\end{abstract}

PACS numbers: 75.45.+j, 03.65.Vf, 05.60.-k, 85.25.-j

Geometric phases arise from adiabatic cyclic evolution in classical and quantum physics [1]. In contrast to dynamic effects, geometric phases depend only on the geometry of the cycle traversed by the state of the system. In parallel transport, for example, a vector is moved along a path without changing its direction in a local coordinate system [1]. For a closed path in a flat Euclidean space, the vector returns exactly to its initial state with respect to a global coordinate system. In a curved space, however, the direction of the vector can change as shown in Fig. 1(a), where the vector moves on a surface of a sphere along a loop enclosing a solid angle $\Omega$. In this case, the angle $\theta$ between the initial and the final state of the vector equals $\Omega$ which depends only on the chosen path, and hence is regarded as a geometric phase. In our universe, geometric phases have been employed for example to measure the curvature of space due to gravitation, and hence to test Einstein's theory of general relativity [2]. This geodetic effect is intended to be measured as changes in the rotation axes of gyroscopes inside a satellite orbiting Earth with the stringent accuracy of $10^{-4}$ in the project Gravity Probe B [3].

We consider adiabatic and cyclic temporal evolution in the ground state of a quantum-mechanical system. The state of any pure quantum system can be described by a complex valued wave function. Thus the simplest geometric phase accumulated in a cycle, i.e., the Berry phase [4], is a phase shift of the complex number multiplying the wave function. As such, the absolute phase of the wave function does not have a physical meaning, and hence is unobservable. Thus the measurements of the Berry phase typically rely on the interference of two states which have undergone a different phase shift $[1,5]$. This technique is also employed in the proposal to measure the Berry phase in an asymmetric superconducting quantum interference device (SQUID) [6]. In phase biased Cooper pair pumps [7] however, the accumulated Berry phase is related to the pumped charge $[8,9]$, and hence we have a fundamentally different way to determine it. Here, we report on the first experimental realization of phase biased Cooper pair pumping in a superconducting loop.

Recently, superconducting circuits have proven to be suitable for coherent manipulations of quantum states $[10,11]$, in particular, two-level quantum systems. The natural scalability of electric circuits makes them potential candidates for qubits, i.e., basic building blocks of the emerging quantum computer. On the other hand, holonomies arising from adiabatic and cyclic evolution in a degenerate eigenspace [12] are unitary transformations which in turn, can be compiled to execute quantum algorithms [13] of practical interest. In particular, the holonomies related to charge transport in Cooper pair devices have been studied theoretically [14]. Thus the observation of the Berry phase in superconducting circuits is an important step towards the development of holonomic quantum computation [15]. To date, holonomic quantum computation has only been demonstrated using liquid state nuclear magnetic resonance [16] (NMR), the scalability of which is limited to about ten qubits.

Single-charge pumping is based on transporting a controlled number $n$ of carriers with quantized charge $e^{*}$ in a cycle repeated at frequency $f$. This principle yields ideally the pumped charge $Q_{p}=n e^{*}$, and hence the pumped current

$$
I_{p}=n e^{*} f .
$$

The carrier charge $e^{*}$ is $e$ for single electrons and $2 e$ for Cooper pairs. In charge pumps described by a phase coherent order parameter field, the phase difference of the field across the device $\varphi$ may play a significant role [7]. In fact for a constant $\varphi$, adiabatic charge pumping gives rise to the Berry phase [8,9]. Interestingly, the accumulated Berry phase, $\Theta_{B}$, in a cycle is related to the pumped charge, $Q_{p}$, by

$$
Q_{p}=-e^{*} \partial \Theta_{B} / \partial \varphi .
$$

In an ideal pumping cycle corresponding to Eq. (1), the 
(a)

(b)
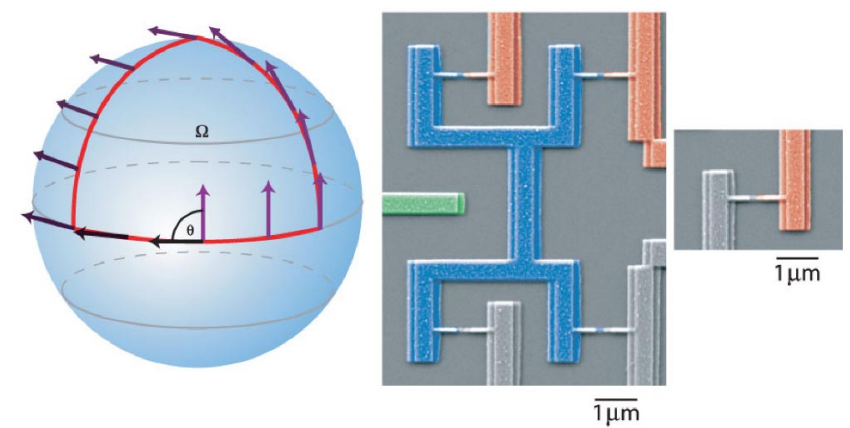

(c)

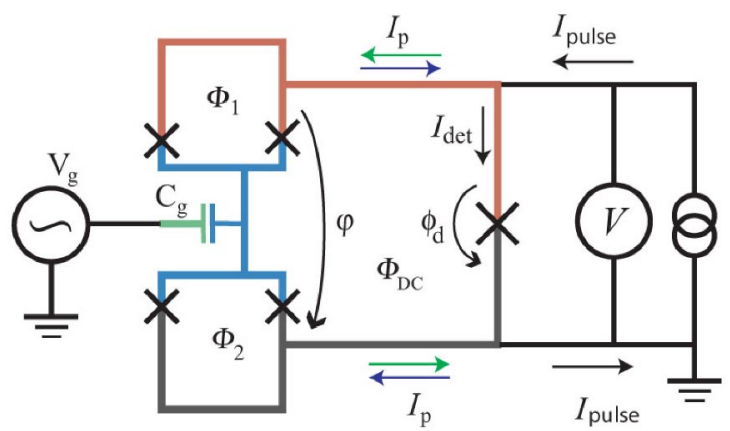

FIG. 1 (color online). (a) Parallel transport of a vector along a path (red line) enclosing a solid angle $\Omega$. The lightest arrow shows the initial state of the vector and the darkest arrow the final state. The resulting angle between the initial and the final vectors $\theta$ is equal to the solid angle $\Omega$. (b) Scanning electron micrograph of the island on the left and of the detector on the right. (c) Simplified circuit diagram of the measured sample. The corresponding parts in the circuit diagram and SEM-images are marked by colors. The independent fluxes $\Phi_{1}, \Phi_{2}$, and $\Phi_{\mathrm{DC}}$ are controlled by on-chip coils [18] and the gate voltage $V_{g}$ is related to the gate charge $n_{g}$ and gate capacitance $C_{g}$ by $n_{g}=$ $C_{g} V_{g} /(2 e)$.

accumulated Berry phase equals $-n \varphi$. However, this kind of pumping does not yield definite fingerprints on the relation between the Berry phase and the pumped charge. Therefore, it is important to reveal the phase coherent nature of pumping from its dependence on $\varphi$. Allowing a nonvanishing average persistent current, i.e., leakage, through the pump during the cycle, the pumped charge becomes phase dependent. In the regime where charge states of the pump are approximately the eigenstates of energy, one obtains in the two-charge-state approximation for a cycle described in Fig. 2(a) $[7,8,17]$

$$
Q_{p} \approx e^{*} n(1-\delta \cos \varphi),
$$

where $\delta \ll 1$ is proportional to the leakage of the pump. Because of its geometric origin, the pumped charge is independent of the pumping frequency. The charge leaked through the pump during a cycle, i.e., the dynamic part of the transferred charge is obtained as

$$
Q_{d} \approx T I_{\mathrm{cmax}} \delta \beta \sin \varphi,
$$

(a)

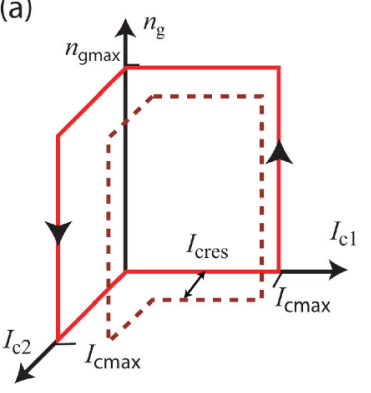

(b)

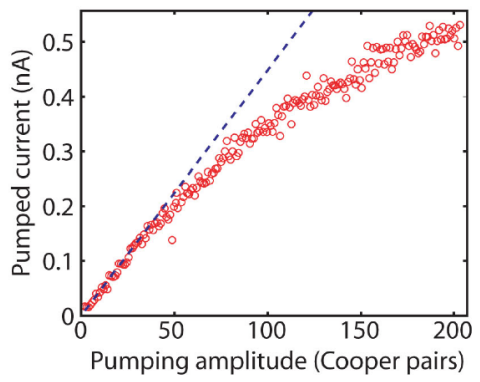

FIG. 2 (color online). (a) Ideal pumping cycle (solid line) in the control parameter space of the sluice. The tunable critical currents of the SQUIDs in the sluice $I_{c 1}=I_{c 1}\left(\Phi_{1}\right)$ and $I_{c 2}=$ $I_{c 2}\left(\Phi_{2}\right)$ are modulated together with the gate charge at the pumping frequency $f=14 \mathrm{MHz}$ for all the data in this paper. In this cycle, the two critical currents are analogous to valves of the charge flow, and the gate charge to a piston. The dashed line illustrates a pumping cycle with finite $I_{\text {cres }}=\delta I_{\text {cmax }} / 2$ introducing finite dynamic current. (b) Pumped current as a function of the maximum gate charge $n_{g}^{\max }$. The blue line shows the theoretical value obtained from Eq. (1) which is expected to be valid in the case of adiabatic pumping for small pumping amplitudes $n_{g}^{\max }$. There are no fitted parameters since the conversion constant of the gate voltage to gate charge is obtained from dc measurements.

where $T=1 / f$ is the period of the pumping cycle, $I_{\text {cmax }}$ is the maximum critical current in the cycle, and $\beta$ is a constant specific to the particular pump and to the control parameter cycle. To justify the validity of the above model in our measurements, we compare the measured pumped current $I_{p}=Q_{p} f$ and the dynamic current $I_{d}=Q_{d} f$ with Eqs. (3) and (4). Here, $I_{d}$ is the supercurrent in the ground state of the system averaged over the pumping cycle and thus it does not depend on $f$ or $n$, nor on the direction of traversing the cycle. On the contrary, $I_{p}$ is proportional to both $f$ and $n$ in the adiabatic evolution, and its sign changes on reversing the path. Agreement with the theory allows us to determine the accumulated Berry phase from Eqs. (2) and (3) as

$$
\Theta_{B} \approx-n(\varphi-\delta \sin \varphi)
$$

Figures 1(b) and 1(c) show the Cooper pair pump, the sluice in a $800 \mu \mathrm{m}^{2}$ superconducting loop with a detector junction. In the ideal pumping cycle shown in Fig. 2(a), an integer number of $n_{g}^{\max }$ excess electron pairs is first attracted to the island through one SQUID and then repelled from the island through the other one using the gate voltage and the tunable critical currents of the SQUIDs in analogy with a piston pump. Hence the sluice generates ideally an average pumped current given by Eq. (1) with $n=n_{g}^{\max }$ The details of the working principle of the sluice can be found in Refs. [8,17].

The Josephson junctions denoted by black crosses in the circuit diagram of Fig. 1(c) consist of $\mathrm{AlO}_{x}$ tunnel barriers fabricated by standard electron beam lithography and two- 
angle evaporation into an all-aluminum device on oxidized silicon wafer. The sluice part of our sample is identical to the one used in Ref. [18] except for up to $10 \%$ smaller junction size and stronger oxidation. The charging energy of the island is difficult to measure in the presence of the detector junction, and hence it is estimated based on the sample used in Ref. [18] to be $2 \mathrm{~K} \times k_{B}$. Using the Ambegaokar-Baratoff formula and the $I V$ characteristics of the sample, the maximum critical currents of the SQUIDs and the detector were estimated to be $30 \mathrm{nA}$ each and $70 \mathrm{nA}$, respectively. Because of the parallel structure of the junctions in the circuit, there is an uncertainty of up to $20 \%$ in the parameter estimation. The plasma frequency of the detector is roughly $20 \mathrm{GHz}$.

We install a Josephson junction working as a threshold current detector in parallel with the sluice forming a superconducting loop as proposed in Ref. [8]. Figure 1(c) also shows our measurement scheme, in which we feed current pulses through the circuit and monitor voltage across it. A voltage pulse is observed if the system switches into the normal state in response to the current pulse. The repetition rate of the measurement was adjusted low enough for the switching events to be uncorrelated. Such a detection method of circulating current was realized, e.g., in the measurements of the superconducting qubit Quantronium [11]. To assure feasible operation, the critical current of the detector is chosen to be much higher than any instantaneous critical current of the sluice. The probability for the system to switch to the normal state depends strongly on the height of the external current pulse $I_{\text {pulse }}$. The switching current $I_{50}$ of the system is defined to be the point where the probability is $50 \%$. We define backward pumping as the direction for which the pumped current adds to the applied $400 \mu$ s current pulse, and hence the current through the detector is given by $I_{\mathrm{det}}=I_{\text {pulse }}+I_{p}-I_{d}$. For forward pumping obtained by traversing the pumping cycle in the opposite direction, the pumped current compensates part of the external pulse: $I_{\mathrm{det}}=I_{\text {pulse }}-I_{p}-I_{d}$. Thus we measure shifts in $I_{50}$ which correspond to twice the pumped current. The average of the two switching currents equals to the dynamic current plus a constant, namely, $I_{\text {det }}$ at the switching point. Note that we restrict our studies to take into account only the dc component of the pumped current at the detector. The effect of the ac component not filtered by the circuit is left for future research.

Figure 2(b) displays the measured pumped current as a function of the gate amplitude for our most ideal pumping cycle. For low enough gate amplitudes, the data shows a nearly linear dependence in good correspondence with the theoretical behavior given in Eq. (1). For high gate amplitudes, the adiabaticity of the pump breaks down and deviation from Eq. (1) is observed. These results demonstrate the first observation of Cooper pair pumping in closed superconducting circuits $[8,19]$. However, the data in Fig. 2(b) does not prove that the current arises from coherent quantum dynamics.
The phase difference $\varphi$ of the superconductor order parameter across the sluice has to be a classical parameter for Eq. (2) to hold. This is satisfied due to the detector junction which protects the sluice from voltage fluctuations, and hence phase biases it. For a detector junction with large enough critical current, the phase difference $\phi_{d}$ (a)

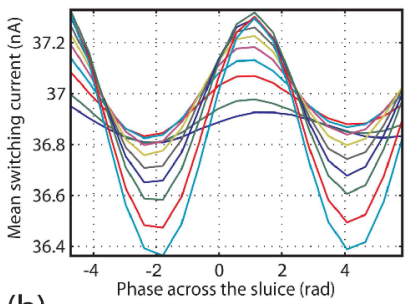

(b)

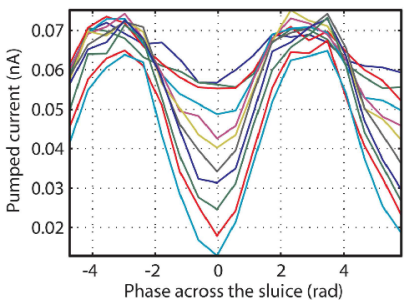

(c)

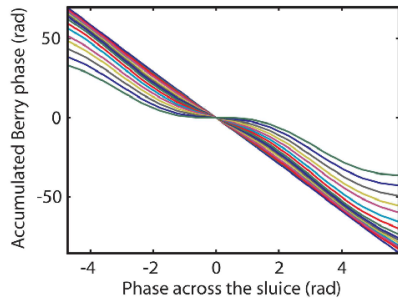

(d)

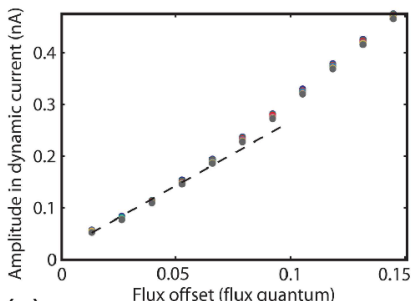

(e)

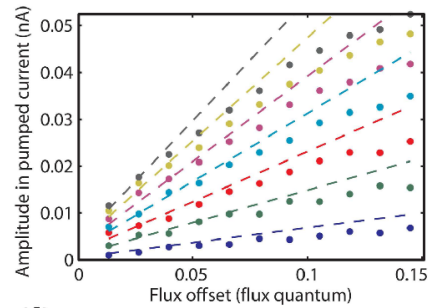

(f)

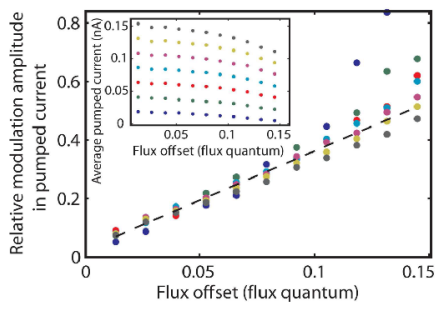

FIG. 3 (color online). (a) Typical switching currents of the system averaged over forward and backward pumping directions as a function of the phase $\varphi$ across the sluice for several residuals of the critical current $I_{\text {cres }}$; see Fig. 2(a). Here, $n_{g}^{\max }=16$. (b) Same as panel (a) but for the pumped current. To obtain the amount of accumulated Berry phase, we make a fit to the data corresponding to Eq. (3) with $\delta$ and $n$ as fitting parameters, the result of which is shown in panel (c). (d) The variation amplitude of the switching current as in panel (a) with respect to the phase across the sluice as a function of the offset in the rf fluxes introducing residual critical current for $n_{g}^{\max }=4$ (blue), 10 (green), 16 (red), 22 (cyan), 28 (magenta), 35 (yellow), and 41 (gray). These values of the maximum gate charge are in the linear adiabatic regime as shown in Fig. 2(b). (e) The variation amplitude of the pumped current as in panel (b) from the same data set as in panel (d). (f) Modulation amplitude in the pumped current divided by the average pumped current (shown in the inset), $\delta$, as a function of the flux offset. The dashed line shows a linear fit to the data for eight smallest offsets, and hence yields the linear dependence of the leakage parameter $\delta$ on the flux offset. The dashed line in panel (d) shows a corresponding theoretical line from Eq. (4) using $\beta=0.0245$ and $I_{\text {cmax }}=$ $30 \mathrm{nA}$. The dashed lines in panel (e) show the linear dependence of $\delta$ multiplied by the average pumped current (see the inset) corresponding to the specific gate amplitude and the smallest flux offset. 
across it is obtained from $\arcsin \left(I_{\mathrm{det}} / I_{c}\right)$ at the switching point where $I_{\text {pulse }}=I_{50}$. Thus we can control $\varphi$ by adjusting magnetic flux $\Phi_{\mathrm{DC}}$ through the loop and using the fundamental phase relation of a superconducting loop $\varphi-$ $\phi_{d}=2 \pi \Phi_{\mathrm{DC}} / \Phi_{0}$, where $\Phi_{0}=h /(2 e)=2.07 \mathrm{fWb}$ is the flux quantum. Because of proper magnetic shielding, the flux offset in $\Phi_{\mathrm{DC}}$ was negligible.

The evidence of the phase coherence in our measurement is shown in Fig. 3. Figure 3(a) presents the variation of the switching current of the system with respect to the phase difference across the sluice controlled by the external flux $\Phi_{\mathrm{DC}}$ as described above, and Fig. 3(b) displays the pumped current from the same measurement. Clear sinusoidal modulation is observed in both curve sets in agreement with Eqs. (3) and (4), implying that the sluice is coherent and phase biased. Note that the minimum of the pumped current corresponds quite accurately to the point of vanishing phase difference across the sluice and the dynamic current is phase shifted by almost $\pi / 2$ radians as in Eqs. (3) and (4). The pumped number of Cooper pairs $n$ and the relative modulation amplitude $\delta$ are determined from the data and the corresponding curves for the accumulated Berry phases are shown in Fig. 3(c) according to Eq. (5). For the largest values of $\delta$, second order corrections to Eq. (5) may modify the estimated value for the Berry phase. We note that this way of measuring the Berry phase is fundamentally different from the conventional method utilizing interference with excited states.

To further test our scheme, we measured the dependence of the modulation amplitudes on the gate amplitude and residual critical current $I_{\text {cres }}=\delta I_{\text {cmax }} / 2$, i.e., leakage, shown in Figs. 3(d) and 3(e). The residual critical current was introduced by an offset in the control fluxes of the SQUIDs from the ideal pumping cycle. Figure 3(f) shows $\delta$ as a function of the offset. As predicted, the modulation amplitude of both, the pumped and the dynamic current, increase with the residual critical current, but only the pumped current depends on the maximum gate charge.

Our observations pave the way for further experiments on Cooper pair pumping in closed circuits [19], on the quantum standard of electric current, for applications of geometric phases in holonomic quantum computation [15], and test the fundamental implications of the quantum theory in an order parameter describing coherent dynamics of a macroscopic number of condensed Cooper pairs. Our measurements are in agreement with a theoretical model neglecting decoherence [20]. On the other hand, the effects of dephasing and dissipation in superconducting qubits are harmful in the manipulation of superconducting quantum systems, see, e.g., Refs. [21]. Thus our results support the robustness of geometric phases against decoherence and fluctuations [22].

We thank the Academy of Finland, Technology Industries of Finland Centennial Foundation, M. M. the Finnish Cultural foundation, Magnus Ehrnrooth
Foundation, and Väisälä foundation for financial support. R. Fazio, F. Hekking, D. Cohen, J. Ankerhold, P. Zanardi, J. Peltonen, A. Niskanen, M. Paalanen, and M. Nakahara are acknowledged for discussions and A. Kemppinen, M. Meschke, O.-P. Saira, and A. Savin for technical support.

Note added. - During the peer review of this work, Leek et al. reported on the observation of the Berry phase in a superconducting qubit [23]. See also recent theoretical work on the Berry phase and Cooper pair pumping [24].

*mikko.mottonen@tkk.fi

[1] Geometric Phases in Physics, edited by A. Shapere and F. Wilczek, (World Scientific, Singapore, 1989); M. Nakahara, Geometry, Topology and Physics (Taylor \& Francis, London, 2003).

[2] I. Ciufolini and E. C. Pavlis, Nature (London) 431, 958 (2004); I. I. Shapiro et al., Phys. Rev. Lett. 61, 2643 (1988).

[3] D. Giulini and N. Straumann, Studies in History and Philosophy of Modern Physics, Vol. 37, p. 115 (2006).

[4] M. V. Berry, Proc. R. Soc. A 392, 45 (1984).

[5] J. Anandan, J. Christian, and K. Wanelik, Am. J. Phys. 65, 180 (1997); Y. Zhang et al., Nature (London) 438, 201 (2005).

[6] G. Falci et al., Nature (London) 407, 355 (2000).

[7] J. P. Pekola et al., Phys. Rev. B 60, R9931 (1999).

[8] M. Möttönen et al., Phys. Rev. B 73, 214523 (2006).

[9] M. Aunola and J. J. Toppari, Phys. Rev. B 68, 020502(R) (2003).

[10] Y. Makhlin, G. Schön, and A. Shnirman, Rev. Mod. Phys. 73, 357 (2001).

[11] D. Vion et al., Science 296, 886 (2002).

[12] F. Wilczek and A. Zee, Phys. Rev. Lett. 52, 2111 (1984); B. Simon, Phys. Rev. Lett. 51, 2167 (1983).

[13] P. Shor, SIAM J. Comput. 26, 1484 (1997); L. K. Grover, Phys. Rev. Lett. 79, 325 (1997).

[14] V. Brosco et al., Phys. Rev. Lett. 100, 027002 (2008); L. Faoro, J. Siewert, and R. Fazio, Phys. Rev. Lett. 90, 028301 (2003).

[15] P. Zanardi and M. Rasetti, Phys. Lett. A 264, 94 (1999).

[16] J. Jones et al., Nature (London) 403, 869 (2000).

[17] A. O. Niskanen, J.P. Pekola, and H. Seppä, Phys. Rev. Lett. 91, 177003 (2003).

[18] J. J. Vartiainen et al., Appl. Phys. Lett. 90, 082102 (2007).

[19] G. Rosenberg and D. Cohen, J. Phys. A 39, 2287 (2006).

[20] See also EPAPS Documents No. E-PRLTAO-100-033818 For more information on EPAPS, see http://www.aip.org/ pubservs/epaps.html.

[21] F. Yoshihara et al., Phys. Rev. Lett. 97, 167001 (2006); J. Martinis et al., Phys. Rev. Lett. 95, 210503 (2005).

[22] R. Fazio, F. W. J. Hekking, and J. P. Pekola, Phys. Rev. B 68, 054510 (2003).

[23] P. J. Leek et al., Science 318, 1889 (2007).

[24] R. Leone and L. Lévy, Phys. Rev. B 77, 064524 (2008). 